\title{
Clustering Approach for Energy Efficient Wireless Sensor Network with Node Mobility
}

\author{
Tejashri Prakash Sawant \\ Department of Information Technology \\ Maharashtra Institute of Technology, Pune
}

\author{
Sumedha Sirsikar \\ Associate Professor \\ Dept. of Information Technology, Pune
}

\begin{abstract}
One of the key challenges in Wireless Sensor Network (WSN) is to minimize energy consumption by each sensor node and increase network lifetime. Sensor network consist of huge number battery powered sensor nodes. It is difficult to recharge these nodes. So, to achieve an appreciable network lifetime, energy consumption of the node should be minimum. By finding the energy efficient route for data transmission network lifetime can be maximized. Cluster based routing protocols are the standard approach for achieving efficient and scalable performance in WSN. Also, there are problem in some approaches such as in static WSN, in which sensor nodes transfer their data to static sink in a multi-hop manner which causes network partition problem known as 'Hot Spot Problem' In case of WSN with mobile sink, as sink travels for data collection from $\mathrm{CHs}$, the load on sink node increases which leads to increase in energy consumption of sink. In this paper a proposed approach is to overcome these problems. The proposed approach uses node mobility to solve these problems. In this approach, mobile sink is used with few mobile nodes. If these mobile nodes are efficiently mobilized then the hot spot problem, as well as sink load problem can be tackled. The movement of mobile nodes is decided on the basis of their distance from CHs. By using node mobility with mobile sink helps to increase the network lifetime and energy efficiency of the network
\end{abstract}

\section{Keywords}

Clustering, Cluster Head, Energy efficiency, Hotspot problem, Node mobility, Network Lifetime, Wireless Sensor Network.

\section{INTRODUCTION}

There are various communication technologies developed in networking, for automatic information collection, computation, and data gathering. The sensor networks have a large number of sensor nodes. They are battery operated. These sensor nodes are massively deployed in a region of interest to collect information from their surroundings. After collecting the information the sensor node do some computation on that data and send it to the sink for further processing. Data transmission from note to note and note to sink should be reliable. So the main objective of WSN is to detect the event and send the information reliably.
Data transmission can be done by using mobile nodes or static nodes. Depending on the application the nodes are taken as mobile or static. For most of the applications sensor nodes and sink remain static for data collection or performing monitoring function in the region of interest. Sensor nodes sense data in the network and forward it to sink over singlehop or a multi-hop manner. The sink collects data from the entire network and sends it for further processing. If the position of a sink is static, a sensor node forwards the data in a multi-hop manner. In this case the nodes near to the sink forward their data as well as the data from other nodes in the network, and consume more energy compared to other nodes in the network. So, nodes near to sink die early and cause network partitioning problem known as 'Hot Spot problem' [1] or 'energy hole problem'. These issues can be solved by making the sink node mobile.

WSN with mobile sink decreases the possibility of network partitioning. In mobile sink network, by moving around the network the sink gather data from sensor nodes. It minimizes the Hot Spot Problem of static WSN. But using only mobile sink it increases load on sink due to this energy consumption by sink increases. To overcome this problem WSN uses few mobile nodes along with mobile sink. Use of few mobile nodes balances load on sink and decreases energy consumption by sink [1]. Also the energy consumption can be minimized by changing the structure of sensor network or the mobility pattern $[2,3]$ or manipulating the data transmission approach. There are various approaches for WSN with mobile nodes and mobile sink. This paper mainly consists of a review of various approaches like LEACH-ME [4,5], VGDRA [6], E2R2 [7,8], MBEENISH and iMBEENISH [9], WRP [10] etc. to provide energy efficiency, efficient network lifetime and throughput. The mobile sink is used along with the static or mobile sensors to enhance the performance of the network.

In recent years WSNs are used for numerous applications such as object tracking, health monitoring [11,12], security surveillance, smart city [13] and intelligent transportation have been proposed. Nowadays everywhere there is monitoring equipment which is deployed with sensors. Smart home environments are yet another application. Many applications in medical, military, etc. field uses Wireless sensor networks like patient monitoring, target tracking, etc 


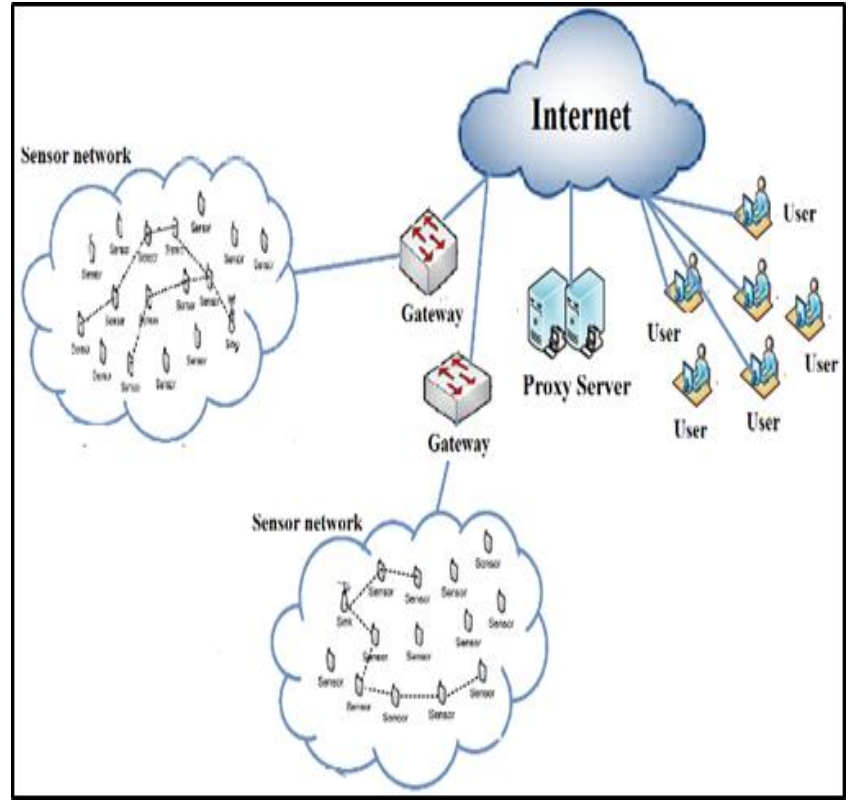

Fig. 1 Wireless Sensor Network

The rest of the paper is ordered as follows: Section 2 contains the Literature Review. Section 3 explains the proposed System Model, Section 4 Contribution followed by section 5 gives result and analysis and section 6 explains the conclusion of the paper.

\section{LITERATURE SURVEY}

The idea of the mobile node in sensor network contains different kinds of routing strategies, protocols and algorithms. The number of papers has shown that the use of some mobile nodes or mobile data collectors as a sink can significantly improve the performance of a network. These protocols show important improvements in WSN. Node mobility with a cluster-based routing protocol, this approach can improve the network in terms of energy conservation and the data transmission. This chapter reviews different approaches that move the sensor node within or around the network area to collect the data from the active sensor nodes based on timedriven and event-driven style.

Kumar Nitesh, Md Azharuddin, Prasanta Jana proposed a Weighted Rendezvous Planning (WRP). In Rendezvous Planning algorithm a Rendezvous design technique is used, where some sensor nodes are selected as an RP's and the nodes which are not RP's will forward data to the nearest RP's. The major issue in this design is to find the set of RP's and determine the tour that visits these RP's. To overcome this issue a Weighted Rendezvous Planning (WRP) protocol is used, where each node is provided with some weight based on the number of packets that it sends and the nearest hop distance. The main aim of this algorithm is to determine the tour for efficient path selection for the movement of mobile sink. WRP selects the sensor nodes as Rendezvous points based on the weight and it prefers the sensor nodes with more weight as a rendezvous point results in a distribution of energy among the sensor nodes uniformly in turn increases the lifetime of the network.

Mariam Akbar, Nadeem Javaid, Muhammad Imran, Naeem Amjad, Majid Iqbal Khan and Mohsen Guizani [9]. In this paper, protocols for heterogeneous wireless sensor networks (WSNs) like BEENISH, improved BEENISH iBEENISH,
MBEENISH, and iMBEENISH are given. BEENISH protocol considers four energy levels of nodes for $\mathrm{CHs}$ selection. Also, it considers residual energy levels of nodes and average energy level of the network. In the iBEENISH protocol, $\mathrm{CHs}$ selection probability varies dynamically in an efficient manner. Through this CHs selection method network lifetime is increased compared to previous protocol. By using a mobile sink in BEENISH (MBEENISH) and iBEENISH (iMBEENISH), the resulting protocols outperform previous protocols regarding stability period, throughput, and network lifetime.

Rajeev Patel, Vikash Porwal, Dr. R. K. Kapoor [4] proposed a $\mathrm{LEACH}-\mathrm{M}$ protocol. It is an advancement to LEACH protocol and M-LEACH protocol in which cluster head is selected on the basis of no movement of a node or it has less relative movement in cluster. It also includes extra time slot in TDMA schedule for mobility calculation based on a number of times node moves from one cluster to another, this extra time slot is added to TDMA schedule on the basis of slot frequency which depends on node movement in the cluster. Here also the node can detect that it goes out of range of current cluster head if it does not receive data request from cluster head in two consecutive frame of data transmission phase and Cluster Head remove the TDMA time slot for mobile node if it does not receive data from a mobile node in two consecutive time slot.

Mobile Sink based Routing Protocol (MSRP) [11] is based on clustering for prolonging the network lifetime. In this protocol, cluster head $(\mathrm{CH})$ collects data from its member nodes and wait for the mobile sink visibility in the region. When the mobile sink is moving in the network, it broadcasts a TDMA schedule to all the nearby $\mathrm{CHs}$ from that region. Each $\mathrm{CH}$ will follow the schedule to forward its data to the mobile sink. Mobile sink visits next $\mathrm{CH}$ based on its residual energy. Mobile sink maintains a record of residual energy of all the $\mathrm{CHs}$. Thus, it moves towards $\mathrm{CH}$ having more residual energy. A mobile sink collects data from $\mathrm{CHs}$ and other neighboring nodes while moving. In this way, the hot-spot problem is eliminated by balancing energy of nodes that results into prolonging the overall network lifetime. However, it does not take into account the latency and the associated packet losses due to sink mobility. This approach causes huge data delivery latency in the parts of the network where some events are occurring most frequently.

\section{SYSTEM MODEL}

In the proposed design, all sensor nodes are deployed into the network. Some nodes will be cluster head and mobile nodes. CHs are selected on the basis of node energy, node degree, and distance from sink. After selection of $\mathrm{CH}$, calculate a number of mobile nodes depending on $\mathrm{CH}$ count. Mobile nodes are selected on the basis of distance from sink and previous mobile nodes as well as node energy. Then cluster formation is done. Member nodes of each cluster will send the data to respective $\mathrm{CH}$. Equal no. of $\mathrm{CH}$ are assigned to mobile nodes and mobile sink. In case of odd no. of $\mathrm{CHs}$, $\mathrm{CHs}$ are assigned equally to mobile nodes and remaining $\mathrm{CHs}$ are assigned to sink. Mobile nodes and mobile sink will collect data from respective CHs. Finally, mobile nodes send data to mobile sink. The proposed system design framework is shown in Fig. 2 


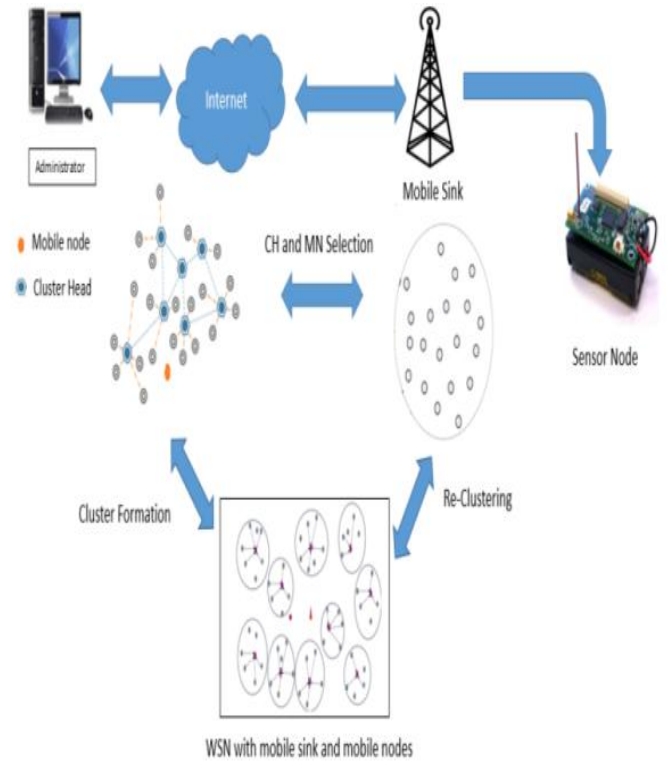

Fig. 2 System Model

\section{CONTRIBUTION}

In this paper, main contribution is to use number of mobile nodes. Sink as well as some nodes are considered to be mobile. Number of mobile nodes is calculated based on $\mathrm{CH}$ count. Selection of mobile nodes is on the basis of node energy and distance from sink. Another modification is done by equally distributing cluster head to MN and MS. This balances load on sink in the network, which reduces the energy consumption by sink. Use of mobile sink with few mobile nodes in the network will increase the network efficiency by reducing the energy consumption of network as well as by increasing the network lifetime. This improves the network performance compare to the previous networking approaches like MSRP in which only mobile sink is used.

\section{RESULTS AND ANALYSIS}

In this section, the result of MSRP and the proposed approached is compared, Graph shows that the improvement in network lifetime and reduced in energy consumption. NS 2 is used for simulation purpose. Graphs are plot for range 250 meters, 300 meters and 350 meters. Nodes are varying from 50 to 120 . Use of mobile nodes with mobile sink shows the better result as compare to MSRP protocol. For energy consumption on $\mathrm{x}$ axis no. of nodes are considered and on $y$-axis energy in Joules are consider. For network lifetime graph on $\mathrm{x}$ axis no. of nodes are considered and on $\mathrm{y}$-axis time in seconds are consider.

Graphs for average energy consumed

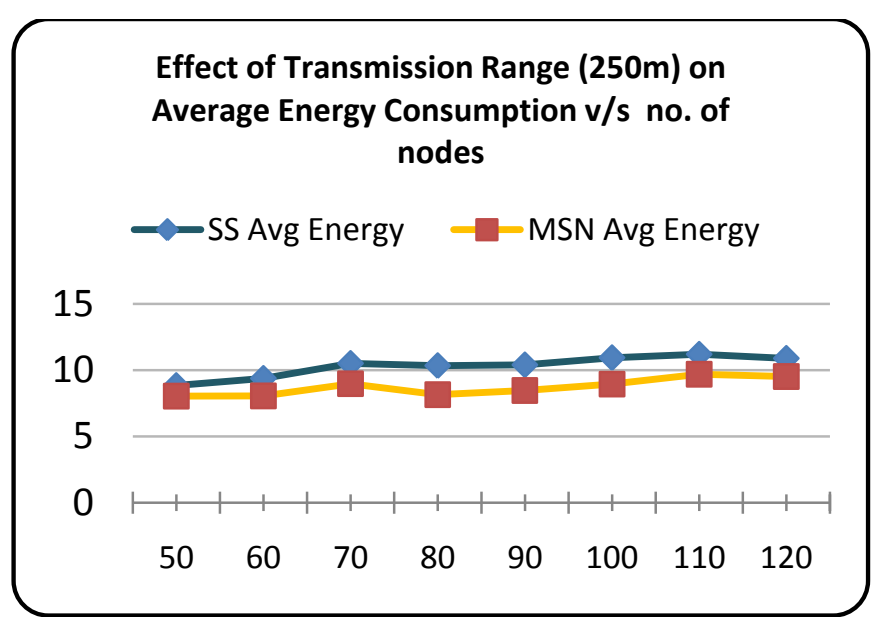

Fig. 3 Graphical representation for Avg. energy consumption vs. No. of nodes for $250 \mathrm{~m}$ range

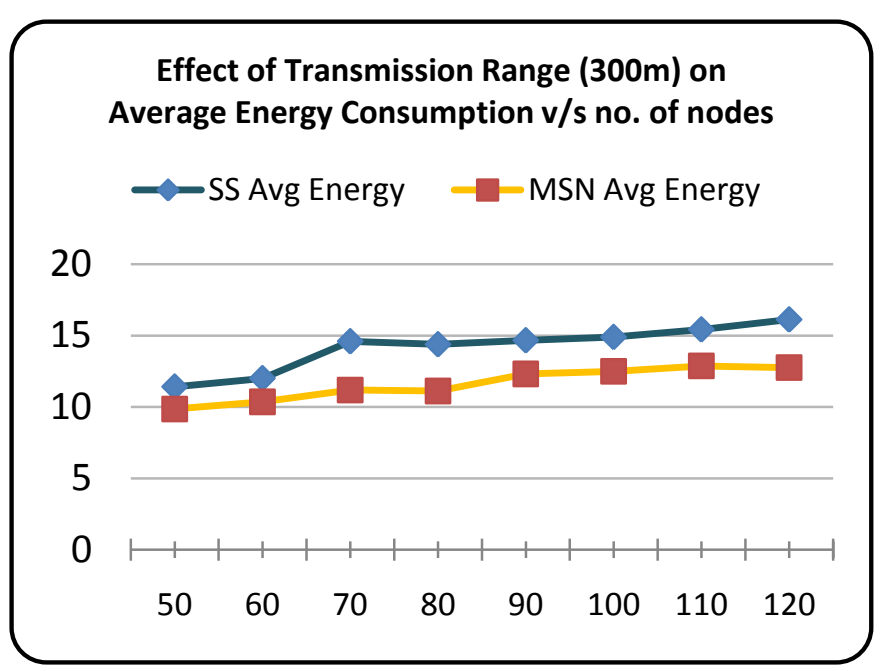

Fig. 4 Graphical representation for Avg. energy consumption vs. No. of nodes for $300 \mathrm{~m}$ range

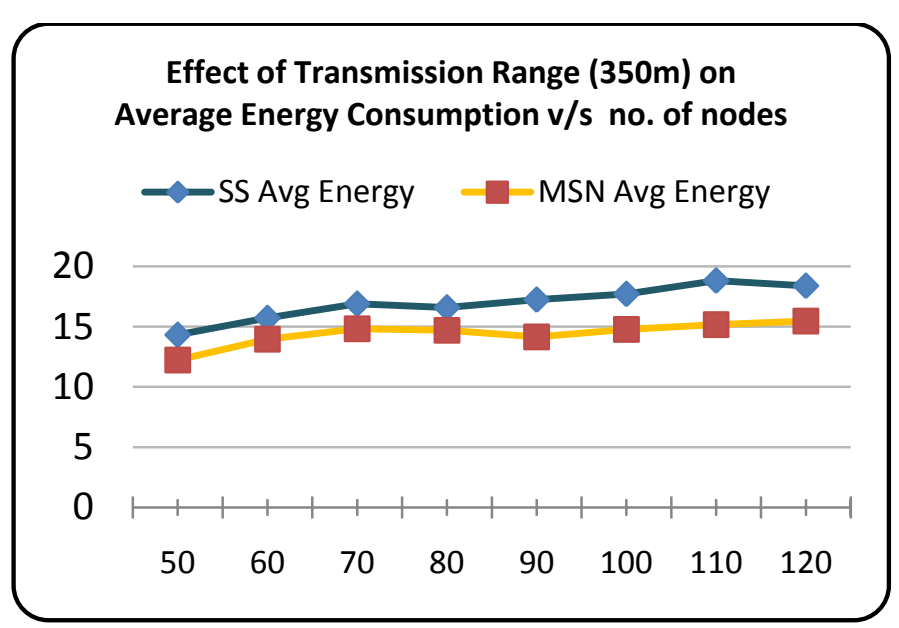

Fig. 5 Graphical representation for Avg. energy consumption vs. No. of nodes for $350 \mathrm{~m}$ range 
Graphs for network lifetime

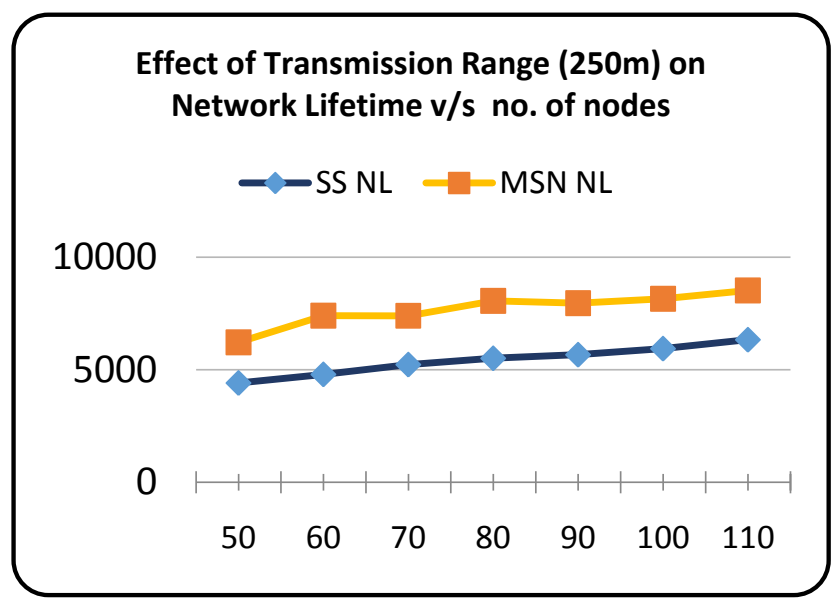

Fig. 6 Graphical representation for Network lifetime vs. No. of nodes for $250 \mathrm{~m}$ range

\section{Effect of Transmission Range $(300 \mathrm{~m})$ on Network Lifetime v/s no. of nodes}
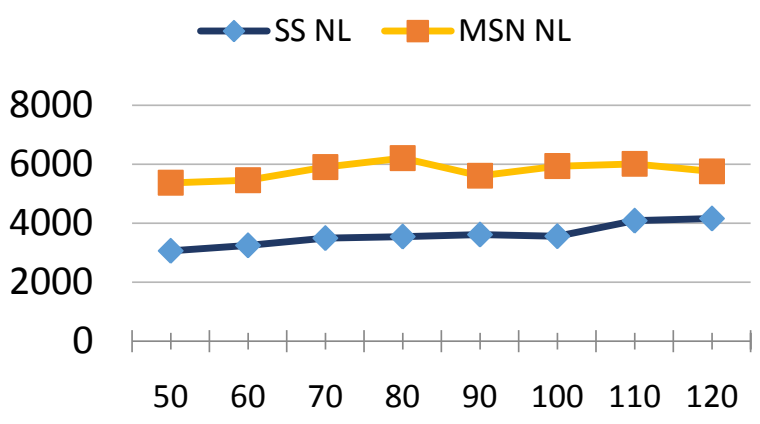

Fig. 7 Graphical representation for Network lifetime vs. No. of nodes for $300 \mathrm{~m}$ range

\section{Effect of Transmission Range (350m) on Network Lifetime $v / s$ no. of nodes}

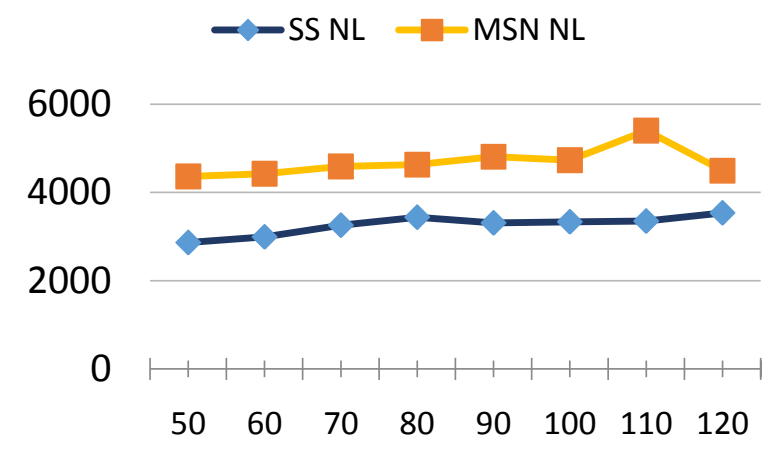

Fig. 8 Graphical representation for Network lifetime vs. No. of nodes for $350 \mathrm{~m}$ range

\section{CONCLUSION}

The energy efficiency is the main issue for wireless sensor network. The previous protocols like MSRP protocol proved to be efficient in terms of a network lifetime and reduced energy consumption. In MSRP only sink is considered as mobile, due to this load on sink increase and energy consumption also increases. So to reduce the load on mobile sink and energy consumption by the sink, mobile nodes are added in the network. In the proposed approach a better solution is provided by using a number of mobile nodes with mobile sink, due to this it improves network lifetime and reduces energy consumption. Also in this network the data collection is done by the mobile sink as well as mobile nodes hence lifetime is increased. So, the proposed approach improves network performance compare to previous approach as shows in the results.

\section{REFERENCES}

[1] Majid I. Khan, Wilfried N. Gansterer, Guenter Haring, 'Static vs. mobile sink: The influence of basic parameters on energy efficiency in wireless sensor networks', IEEE, 2013

[2] Chenglin Zhao, Yongxing Wang, Xuebin Sun and Ting Jiang, "An Energy Saving Cluster Routing for Wireless Sensor Network with Mobile Sink," International Conference on Advanced Intelligence and Awareness Internet, pp 113-117,2010

[3] M. I. Khan, Wilfried. Gansterer and Guenter Haring, "Static vs. mobile sink: The influence of basic parameters on energy efficiency in wireless sensor networks," Elsevier Computer Communications, November 2012

[4] Renugadevi G., Sumithra M.G., "An Analysis on LEACH-Mobile Protocol for Mobile Wireless Sensor Networks," International Journal of Computer Applications (0975 - 8887) Volume 65- No.21, March 2013

[5] Rajanpreet Bhatti, Gurinderjeet Kaur, "Virtual Grid Based Energy Efficient Mobile sink Routing Algorithm for WSN", IEEE 2017

[6] Hiren Kumar Deva Sarma, Rajib Mall, Avijit Kar, "E2R2: Energy-Efficient and Reliable Routing for Mobile Wireless Sensor Networks", 1932-8184 (C) 2015 IEEE.

[7] Hiran Kumar Deva Sarma, Avijit Kar, Rajib Mall, "Energy Efficient and Reliable Routing for Mobile Wireless Sensor Networks.” IEEE 2010, pp. 1-6

[8] Mariam Akbar, Nadeem Javaid, Muhammad Imran, Naeem Amjad, Majid Iqbal Khan and Mohsen Guizani, "Sink mobility aware energy-efficient network integrated super heterogeneous protocol for WSNs", 2016 Springer

[9] Wan Aida Nadia binti Wan Abdullah, N. Yaakob, R Badlishah, A Amir, Siti Asilah binti Yah, "On the effectiveness of Congestion Control Mechanisms for Remote Healthcare Monitoring System in IoT Environment - A Review", 2016 3rd International Conference on Electronic Design (ICED), August 11-12, 2016, Phuket, Thailand

[10] Kumar Nitesh, Md Azharuddin, Prasanta Jana, "Minimum spanning tree-based delay-aware mobile sink traversal in wireless sensor networks", International Journal of Communication Systems, pp. e3270, 2017, ISSN 10745351 
[11] Suwarna Latambale, Sumedha Sirsikar, "A Survey of various Sink Mobility based Techniques in Wireless Sensor Network", Indore, India (C) 2016 ACM. ISBN 978-1-4503-4278-0/16/03

[12] Wan Aida Nadia binti Wan Abdullah, N. Yaakob, R Badlishah, A Amir, Siti Asilah binti Yah, "On the effectiveness of Congestion Control Mechanisms for Remote Healthcare Monitoring System in IoT
Environment - A Review", 2016 3rd International Conference on Electronic Design (ICED), August 11-12, 2016, Phuket, Thailand

[13] Suganya E, Vijayashaarathi S, "Smart Vehicle Monitoring System for Air Pollution Detection using Wsn", International Conference on Communication and Signal Processing, April 6-8, 2016, India 